REVIEW

\title{
Urinary tract infections: new insights into a common problem
}

\author{
R Kucheria, P Dasgupta, S H Sacks, M S Khan, N S Sheerin
}

Postgrad Med J 2005;81:83-86. doi: 10.1136/pgmi.2004.023036

This review discusses recent advances in the understanding of how the common pathogen, uropathogenic Escherichia coli, interacts with the host to lead to infection.

See end of article for authors' affiliations

.....................

Correspondence to: Mr R Kucheria,

Department of Urology, 1 st Floor Thomas Guy House, Guy's Hospital, London SE1 9RT, UK; rkucheria@ hotmail.com

Submitted 26 April 2004 Accepted 14 July 2004
U rinary tract infection (UTI) is one of the most common bacterial infections encountered in clinical practice in Europe and North America. It is estimated that 150 million cases of UTI occur on a global basis per year resulting in more than 4 billion pounds ( 6 billion dollars) in direct health care expenditure. ${ }^{1}$ Young, otherwise healthy, women are commonly affected with an estimated incidence of 0.5-0.7 infections per year. ${ }^{2}$ Of the women affected $25 \%-30 \%$ will go on to develop recurrent infections not related to any functional or anatomical urinary tract abnormality. Although uncomplicated infections do not result in long term sequelae, for example renal scarring, they cause significant morbidity, particularly when recurrent.

There have been important advances in defining the bacterial virulence factors that increase bacterial infectivity. Other investigators have studied factors that lead to host susceptibility. However, it is the complex interaction between these bacterial virulence factors and the host response that determines the outcome of bacterial exposure. Advances in cellular and molecular biology have improved our understanding of the bacteria-host association, nevertheless, it remains incompletely understood.

Uropathogenic Escherichia coli (UPEC) is the causative agent in $70 \%-95 \%$ of community acquired UTI and $50 \%$ of all cases of nosocomial infection. In this review we will discuss recent advances in our understanding of how this common pathogen interacts with the host to lead to infection and subsequent resolution or, in a minority, repeated infection

\section{VIRULENCE FACTORS}

Uropathogenic bacteria have evolved a range of virulence factors that promote colonisation and infection of the urinary tract. The virulence factors most commonly associated with these organisms include possession of fimbriae with adhesin tips, protectins, bacterial capsule including lipopolysaccharide (LPS), and production of toxins such as haemolysin and colony necrotising factor.

\section{Fimbriae (Pili)}

Fimbriae are thin, hair-like, surface adhesive organelles made of protein subunits. A number of different types have been described, which are distinguished by their size (length and diameter) and their host target molecule. They were first described in the 1960s by J P Duguid, Professor of Microbiology at the University of Dundee and are typically associated with Gram negative bacteria such as $E$ coli and Salmonella spp. Fimbriae originate in the cytoplasm of the cell and project through the cell membrane and the cell wall. They bind glycoprotein or glycolipid moieties on urothelial cells allowing the bacteria to attach to the epithelium and persist within the urinary tract. The most common fimbrial proteins associated with UPEC infection are type 1 , $\mathrm{P}$, and Dr fimbriae.

Type 1 fimbriae

Type 1 fimbriae, the single most commonly expressed virulence factor, are produced by more than $80 \%$ of all UPEC. Electron microscopy and biochemical studies have suggested that these bacterial cell wall projections bind to the urothelial mannosylated glycoproteins uroplakin Ia and Ib via the adhesin subunit FimH, located at the fimbrial tip. They are also thought to be responsible for increasing the inflammatory response associated with bacterial adhesion and invasion. Studies have shown specific receptors on mast cells that bind to the FimH tip to initiate an inflammatory response by releasing inflammatory mediators. ${ }^{3}$ Tamm-Horsfall protein (THP), synthesised in the distal nephron, also binds type 1 fimbriae, preventing adhesion and is therefore uroprotective.

\section{Type P fimbriae}

Type P fimbriae are the second most common virulence factor of UPEC. They consist of heteropolymeric fibres composed of different protein subunits, encoded by the pap A-K gene operon. ${ }^{4}$ These fimbriae recognise kidney glycosphingolipids carrying the Gal $\alpha(1-4)$ Gal determinant via its papG adhesin. They are important in the pathogenesis of ascending UTI and pyelonephritis in humans and have been identified in $80 \%$ of pyelonephritic $E$ coli isolates. ${ }^{5}$ In experimental pyelonephritis, $\mathrm{P}$ fimbriae are as important as vesico-ureteric reflux in the development of ascending infection. ${ }^{6} \mathrm{P}$ fimbriae mediated attachment to the oligosaccharide receptors also induces epithelial cell activation and the production of cytokines such as IL6 and IL8 in vitro. ${ }^{7}$

Abbreviations: UTI, urinary tract infection; UPEC, uropathogenic Escherichia coli; LPS, lipopolysaccharide; CNF, cytotoxic necrotising factor; THP, Tamm-Horsfall protein; TLR, toll-like receptor; MAC, membrane attack complex 


\section{Type Dr fimbriae}

This family of adhesins are a commonly encountered virulence factor. $E$ coli strains expressing Dr fimbriae are able to bind to and possibly invade epithelial cells by interacting with a complement regulatory protein, decay accelerating factor (DAF). E coli strains, which express adhesins of the Dr family, are associated with cystitis $(30 \%-50 \%)$ in children and pregnancy associated pyelonephritis $(30 \%){ }^{8}$

\section{Siderophores}

Iron is an essential nutrient for bacterial viability within the host and UPEC exhibit multiple mechanisms for iron extraction, the most common being siderophore-siderophore receptor systems and heme uptake. ${ }^{9}$ The siderophore systems include entrobactin and aerobactin with receptors such as IreA and IroN and Iha receptors. Iha receptors have also been shown to have adherence properties therefore aiding the virulence further.

\section{Toxins}

\section{Haemolysins}

There are two common types of this toxin, $\alpha$-haemolysin and $\beta$-haemolysin. $\alpha$-Haemolysin is commonly produced by strains isolated from cases of human UTI and other extra intestinal infections. It is a heat-labile extracellular protein and its production can be plasmid or chromosomally determined. ${ }^{10}$

It is a pore forming cytolysin, and lyses erythrocytes by causing dissipation of transmembrane ion gradients. Iron, is released from cells that can be used by the bacteria for propagation via the siderophore systems. Water moves into the cell as a result of the increased intracellular osmotic pressure, causing the cell to swell and rupture. $\beta$-haemolysin is a cell associated haemolysin with a similar range of haemolytic activity as $\alpha$-haemolysin.

\section{Cytotoxic necrotising factor (CNF)}

CNF types 1 and 2 belong to a group of bacterial toxins that modify Rho, a subfamily of small GTP binding proteins that are regulators of the actin cytoskeleton. Eukaryotic cells exposed to CNFl exhibit membrane ruffling, formation of focal adhesions and actin stress fibres, and DNA replication in the absence of cell division, resulting in the formation of enlarged multinucleated cells. Even though, it has been shown in vitro that CNF causes cell lysis and hence contributes to virulence, the direct proof of the toxin's role in disease remains to be determined.

\section{Protectins}

UPEC express a covering of group 2 or 3 polysaccharide capsule and LPS as compared with group 1 capsules of the commensals. The polysaccharide, especially LPS, plays an important part in virulence by activating various signalling pathways inducing an immune response from the host. The details of this cascade are discussed later in this review.

\section{HOST FACTORS}

Host factors play a vital part in the balance between sterility and infection of the urinary tract. Most of the pathogenic bacteria that cause UTI are from the host's own bowel flora and enter the bladder via the urethra.

Host factors can be divided into: (a) natural host defence factors, and (b) acquired host factors (host response) that are activated in response to bacterial invasion.

\section{Natural host defences}

\section{General defence factors}

The urinary tract has a number of specialised defences against bacterial colonisation, keeping the urine sterile. These include the unidirectional flow of urine in a peristaltic fashion from the kidney to the bladder. In the bladder, secretion of glycosamines by bladder transitional cells prevents bacterial adherence by forming a mucin layer. Low $\mathrm{pH}$ and presence of salts and urea reduce bacterial survival within the urinary tract.

\section{Tamm-Horsfall protein}

THP, also known as uromucoid, is a glycoprotein that is synthesised exclusively by the epithelial cells of the thick ascending loop of Henle and early segment of distal convoluted tubules. This acts as an antiadherence factor by binding to UPEC expressing type 1 fimbriae and forming an uromucoid-coliform complex, which is then cleared by voiding. However, a recent study has shown that at concentrations below $30 \mu \mathrm{g} / \mathrm{ml}$, THP stimulates bacterial adherence.

\section{Defensins}

The defensins are a group of small highly cationic antimicrobial peptides produced by a variety of mammalian cells. They can divided into three groups based on their molecular structure, namely $\alpha, \beta$, and $\theta$. The first two are found in human cells including macrophages, neutrophils, and epithelial cells lining the bronchial tree, gastrointestinal tract, and the renal tract. In the urinary tract, they are produced after exposure to pathogens and have the capacity to kill bacteria, fungi, and some encapsulated viruses. Defensins attach to the anionic phospholipids on the cell wall of organisms and disrupt their cell membrane function, increasing cell permeability and causing cell death. They have also been shown to augment innate responses by causing mast cell degranulation and promoting neutrophil chemotaxis by increasing IL8 production. ${ }^{11}$

\section{P blood group secretor status}

P fimbrial attachment of bacteria to the urothelium occurs via glycolipid epithelial cell receptors. These receptors are members of the $\mathrm{P}$ blood group, including several antigens, $\mathrm{P}_{1}$, $\mathrm{P}$, and Pk. P fimbriae have been shown to haemagglutinate erythrocytes from people who have the $\mathrm{P}_{1}$ blood group antigen. If this antigen is secreted (secretor positive), there is a reduced incidence of bacterial adherence and thus UTI. ${ }^{12}$

Additionally, urine contains microbicidal proteins including an enzyme called lysozyme that specifically cleaves the cell wall of Gram positive bacteria. Another specialised defence against bacterial invasion and colonisation is bacterial internalisation by epithelial cells, a rapid apoptosis-like process involving DNA fragmentation and activation of proteolytic enzymes and cell shedding, thereby removing bacteria from the renal tract.

\section{HOST IMMUNE RESPONSE}

Once the bacteria come into contact with urothelial cells, an innate immune response is started. This occurs via the interaction between bacterial factors, for example, fimbriae and LPS, and the epithelial receptors leading to the activation of a variety of signalling pathways.

LPS is an integral component of the cell wall of Gram negative bacteria and a potent activator of various cell types. LPS stimulates cells of innate immunity via the interaction with toll-like receptor 4 (TLR4). TLRs are a recently described family of pattern recognition receptors expressed on leucocytes and epithelial cells, which recognise preserved molecular motifs found on pathogens. At present 10 mammalian TLRs have been identified (TLRI-TLR10) that interact with different components of invading organisms. For example, TLR2 has been shown to respond with components of Gram positive bacteria while TLR4 responds to Gram negative bacteria.

For LPS to activate cells via TLR4, a number of accessory molecules are required. These include LPS binding protein (LBP), CD14, and MD2, and it is thought that these molecules form a receptor complex on the cell surface. This signals the activation of intracellular signalling pathway 
leading to release of cytokines such as tumour necrosis factors (TNF), interferon $\gamma$, IL8, and ILl. The importance of TLR4 is shown in a mouse strain that has defective LPS signalling caused by mutations in the TLR4 gene. This strain is unable to efficiently clear bacteria from the bladder after infection with UPEC. ${ }^{13}$ It also activates the coagulation cascade and the complement cascade initiating an immune response.

\section{Complement activation}

The activation of complement cascade occurs via three distinct pathways, namely, the classic, alternative, and mannan binding lectin (MBL pathways), all of which can be activated by invading organisms. Activation by any of these pathways leads to formation of C3 and C5 convertases and the production of biologically active components including anaphylatoxins, opsonins, and membrane attack complex (MAC). The anaphylatoxins, C3a and C5a, act by attracting and activating neutrophils and macrophages, while the opsonins, C3b, C4b increase phagocytosis of bacteria. The MAC binds to the lipid bilayer of the bacterial cells, creating pores in the cell surface through which solutes can diffuse, leading to osmotic lysis of the bacterium. The complement system can also augment the adaptive immune response. For example, C3d has been shown to increase sensitivity of the immune response via opsonisation of antigen and subsequent interaction with complement receptor 2 (CR2) on immune cells.

\section{Adaptive immunity}

Despite the innate immune response, the bacteria still can persist in the urinary tract. Therefore a more specific adaptive immune response ensues, which includes both humoral and cellular responses. Even though serum production of antibodies is characteristically difficult to detect in UTI, local production of antibodies has been reported from the bladder and urethra. ${ }^{14}$ It has been postulated that the sensitised B lymphocyte cells migrate to the lamina propria from the lymphatics and differentiate into IgA secreting cells. ${ }^{15}$ The role of these secretory IgA ( $\operatorname{IgA}$ ) antibodies in UTIs is poorly understood. However, it has been suggested that these antibodies inhibit bacterial colonisation by lowering the bacterial adherence to the mucosa. ${ }^{16}$

\section{BACTERIAL PERSISTENCE}

Despite numerous host defences and even antibiotic treatments that can effectively sterilise the urine, a significant proportion of patients end up having recurrent UTI. Current knowledge of the pathogenesis of UTI cannot explain the recurrence of infection despite repeated and long term use of antimicrobial treatment. The UPEC are thought to colonise in the gastrointestinal tract, from where they migrate to the periurethral area and ascend up the urinary tract to cause infection. Furthermore, women who suffer from recurrent UTI have a higher rate of vaginal colonisation with UPEC. However, application of antibiotic ointment to the periurethral area does not reduce the risk of UTI. ${ }^{17}$

Recent studies show that uropathogens can invade the superficial epithelial cells and replicate, forming large foci of intracellular $E$ coli. The invasion of bladder epithelial cells triggers the immune host response and causes the superficial cells to exfoliate. The exfoliation is considered to be an effective defence mechanism by the host. However, before the completion of this process the intracellular bacteria reemerge from the host cells and invade the underlying epithelial cells (the new superficial layer) and consequently persist within the urinary tract. In this manner, the UPEC may continue to reinvade the epithelium establishing a persistent, quiescent bacterial reservoir within the bladder mucosa, or cause recurrent bladder infections. The existence of such reservoirs within the urinary tract may help explain the recurrent nature of UTIs. ${ }^{18}$

In an experimental mouse model, bacteria have been shown to use host immune responses such as complement activation to its advantage in invading the host cells. Springall et al discovered that mice deficient in C3 gene, when compared with wild mice, had a significantly lower rate of infection upon innoculation with UPEC in the urinary bladder. Opsonisation of bacteria with $\mathrm{C} 3$ was shown to assist in their invasion of the urothelial cells, possibly via interaction with epithelial cell surface receptors, however, these are yet to be identified. Such adaptations bring to light the fact that bacteria are ever evolving to maintain their virulence. ${ }^{19}$

\section{CLINICAL ASPECTS}

It is believed that $10 \%-20 \%$ of women with UTI have recurrent episodes. The risk factors include both genetic and behavioural factors. Women with recurrent infection have an increased occurrence of recurrent UTI in their first degree female relatives. ${ }^{20}$ The commonest behavioural factor associated with recurrent UTI is use of spermicides as a contraceptive measure.

The European urology guidelines recommend managing recurrent UTI using antibiotics in two different regimens. The first is long term low dose prophylaxis and the other is self start treatment. The second regimen has been used effectively as post-coital prophylaxis for women who suffer from intercourse associated recurrent UTI. Although there are numerous antibiotics used worldwide the common ones include co-trimoxazole, nitrofurantoin, ciprofloxacin, and cephalosporins.

\section{Cranberry juice}

Complementary therapy for this condition is also popular although the evidence is patchy. The commonest in use is cranberry juice and products. The Cochrane review on the use of cranberry juice in the prevention of UTI suggested there is some evidence from two good quality randomised control trials that cranberry juice may decrease the number of symptomatic UTI over a 12 month period in women. ${ }^{21}$ It has been shown that cranberries prevent bacteria (particularly $E$ coli) from adhering to urothelial cells of the bladder. Cranberries contain two compounds that inhibit adherencefructose and a polymeric compound of unknown nature. Although many juices contain fructose, only cranberries and blueberries contain the polymeric compound.

\section{Probiotics}

The other product being increasingly used is probiotics or "the friendly bacteria". The commonly used or marketed are the lactobacilli species. Initial trials with oral probiotics did not show convincing results. However, Reid et $a^{22}$ showed $43 \%$ reduction in incidence of UTI in women with history of recurrent UTI by using hydrogen peroxide producing $10^{8-9} \mathrm{~L}$ acidophilus in a product called Vivag, twice daily for six days. The lactobacilli were delivered in a tampon vaginally. Although these findings are encouraging there is still the need to continue research in improving its efficacy and specificity before it can be adopted as a viable choice for prophylaxis.

\section{Oestrogen supplement}

In postmenopausal women it has been shown that the lack of oestrogen makes the vaginal more prone to pathogenic bacterial colonisation. Therefore, trials with topical oestrogen supplements have been performed on both sides of the Atlantic, but, with mixed results. Raz et al studied 93 postmenopausal women with a history of recurrent UTI in a randomised, double blind, placebo controlled trial of a 
topically applied intravaginal oestriol cream and evaluated patients monthly for eight months. ${ }^{23}$ The incidence of UTI in the group given oestriol was significantly reduced compared with that in the placebo group (0.5 compared with 5.9 episodes per patient year, $\mathrm{p}<0.001$ ). Lactobacilli were absent in all vaginal cultures before treatment and reappeared after one month in $61 \%$ of the 36 oestriol treated women compared with none of 24 placebo recipients $(p<0.001)$. In a more recent randomised control trial by Sobel ${ }^{24}$ the results were not as expected. Data collected during the study showed that oestriol released from the vaginal pessary did achieve increased maturation of vaginal epithelial cells. However, in contrast with previous studies, this form of oestrogen administration failed to achieve its functional goal because $\mathrm{pH}$ remained abnormally high ( $\mathrm{pH}$ was 5.3), and a lactobacillus dominant vaginal flora was not achieved.

Even though these novel treatments raise our expectations, none of them seem to be convincing enough to be used in the clinical settings to prevent recurrent UTI.

\section{FUTURE PROSPECTS}

Despite many advances in our understanding of the interaction between pathogenic bacteria and the host renal tract many aspects of the pathogenesis of UTI are still poorly defined. Ever improving scientific techniques allow us to study this interaction at a molecular and cellular level and will undoubtedly provide insight into the mechanisms underlying this common disease. In addition as our understanding improves we may be able to target treatments to prevent infection that do not rely on conventional antibiotics, with the increasing problems of bacterial resistance. Several areas of particular interest exist.

Genomic and proteomic studies of host and pathogens show promise in finding new strategies to study hostpathogen interactions and develop new antimicrobial agents. We now have human and bacterial genome sequences that may identify new genes involved in infection. We can study the level of expression of large number of genes during infection with techniques such as serial analysis of gene expression (SAGE), oligonucleotide microarray "chips", and DNA microarrays. Microarrays use primary sequence data to measure transcript levels and detect sequence polymorphisms for thousands of genes simultaneously. Therefore, the molecular details of the complex interaction between a pathogen and host can be studied to identify virulence associated microbial genes and host defence strategies and in particular polymorphisms in these responses.

\section{Vaccines}

Because of the uncertainty regarding the importance of the adaptive immune response in preventing UTI the role of vaccination has been unclear. However, as our understanding of the molecular aspects of bacterial binding has increased it has been possible to design vaccines that target adaptive responses against specific bacterial proteins and functions. The antibodies produced can interfere with the function of essential bacterial virulence factors and can prevent the bacteria from adhering to and invading the host epithelium. One such target is Fim H tip adhesin of type 1 fimbriae. Intravesical inoculation of monkeys with Fim $\mathrm{H}$ protected them from further experimental UTI. ${ }^{25}$ Similarly, inoculation of P fimbriae adhesion PapG (Pap DG vaccine) has been shown to defend against recurrent UTI in a monkey model. ${ }^{26}$ The increasing importance of intracellular bacteria in UTI pathogenesis suggests that blocking internalisation might be an effective way to stop recurrent UTI.

As it is clear that the inflammatory response causes injury and scarring of the urinary tract (as well as other affected tissues), changing it may reduce or prevent irreversible damage to tissue hence reducing the associated morbidity of UTI. Neutrophil activation and the complement cascade and its receptors may be potential therapeutic targets.

\section{Authors' affiliations}

R Kucheria, P Dasgupta, M S Khan, Department of Urology, Guy's and St Thomas' Hospital NHS Trust, London, UK

N S Sheerin, S H Sacks, Department of Renal Medicine and Transplantation, King's College London, Guy's Hospital

Funding: Wellcome Trust (Neil S Sheerin).

Conflicts of interest: none declared.

\section{REFERENCES}

1 Harding GKM, Ronald AR. The management of urinary infections: what we have learned in the past decade. International Journal of Antimicrobial Agents 1994:4:83-8

2 Hooton TM, Scholes D, Hughes JP, et al. A prospective study of risk factors for symptomatic urinary tract infection in young women. N Engl J Med 1996;335:468-74.

3 Abraham SN, Shin JS, Malaviya R. Type 1 Fimbriated Escherichia coli-mast cell interactions in cystitis. J Infect Dis 2001;183:S51-5.

4 Hull RA Gill RE, Hsu P, et al. Construction and expression of recombinant plasmids encoding type-1 or D mannose-resistant pili from a urinary tract infection Escherichia coli isolate. Infect Immun 1981;33:933-8.

5 Plos K, Connell H, Jodal U. Intestinal carriage of P fimbriated Escherichia coli and the susceptibility to urinary tract infection in young children. $J$ Infect Dis 1995; 171:625-31.

6 Roberts JA, Suarez GM, Kaack B, et al. Experimental pyelonephritis in the monkey. VII. Ascending pyelonephritis in the absence of vesicouretral reflux. $J$ Urol 1985; 133:1068-75

7 Hedges SR, Agace WW, Svanborg C. Epithelial cytokine responses and mucosal cytokine networks. Trends Microbiol 1995;3:266-70.

8 Goluszko P, Moseley SL, Truong LD, et al. Development of experimental model of chronic pyelonephritis with Escherichia coli O75:K5:H-bearing Dr fimbriae: mutation in the dra region prevented tubulointerstitial nephritis. J Clin Invest 1997:99: 1662-72.

9 Russo TA, Carlino UB, Johnson JR. Identification of ire A, a novel iron regulated virulence gene in an extraintestinal pathogenic isolate of Escherichia coli. Infect Immun 2001;69:6209-16.

10 Menestrina G, Pederzolli C, Dalla Serra M, et al. Permeability increase induced by Escherichia coli Hemolysin $A$ in human macrophages is due to the formation of ionic pores: a patch clamp characterization. J Membr Biol 1996; 149:113-21.

11 Ganz T. Defensins: Antimicrobial peptides of innate immunity. Nature Rev Immunol 2003;3:710-20.

12 Lomberg $\mathbf{H}$, Hanson LA, Jacobsson $B$, et al. Correlation of $\mathrm{P}$ blood group, vesicoureteral reflux, and bacterial attachment in patients with recurrent pyelonephritis. N Engl J Med 1983;308:1 189-92.

13 Hagberg L, Hull R, Hull S, et al. Difference in susceptibility to gram-negative urinary tract infection between $\mathrm{C} 3 \mathrm{H} / \mathrm{HeJ}$ and $\mathrm{C} 3 \mathrm{H} / \mathrm{HeN}$ mice. Infect Immun 1984:46:839-44.

14 Kuriyama M. The study of urinary secretory lgA. (I). Its localisation in the urinary tract. (Author's translation). Nippon Hinyokika Gakkai Zasshi 1979;70:602-7.

15 Underdown BJ, Schiff JM. Immunoglobulin A: strategic defence initiative at the mucosal surface. Annu Rev Immunol 1986;4:389-417.

16 Svanborg C, Svennerholm AM. Secretory immunoglobulin A and G antibodies prevent adhesion of Escherichia coli to human urinary tract epithelial cells. Infect Immun 1978;22:790-7.

17 Cass AS, Ireland GW, Aal C. Antibacterial perineal washing for prevention of recurrent urinary tract infections. Urology 1985;25:492-4.

18 Mulvey MA, Joel SD, Hultgren SJ. Establishment of a persistent Escherichia coli reservoir during the acute phase of a bladder infection. Infect Immun 2001;69:4572-9.

19 Springall T, Sheerin NS, Abe K, et al. Epithelial secretion of C3 promotes colonization of the upper urinary tract by Escherichia coli. Nat Med 2001;7:803-8

20 Fenell RS, Wilson SG, Garin EH, et al. Bacteriuria in families of girls with recurrent bacteriuria. Clin Paediatr 1977;16:1132.

21 Jepson RG, Mihaljevic L, Craig J. Cranberries for preventing urinary tract infections. Cochrane Library. Issue 2. Chichester: Wiley, 2004.

22 Reid G, Bruce AW. Urogenital infections in women: can probiotics help? Postgrad Med J 2003;79:428-32.

23 Raz R, Stamm WE. A controlled trial of intravaginal estriol in postmenopausal women with recurrent urinary tract infections. N Engl J Med 1993;329:753-6.

24 Sobel JD. Estrogen replacement therapy to prevent recurrent urinary tract infection in postmenopausal women. Current Infectious Disease Reports 2003;5:479-80

25 Langermann S, Mollby R, Burlein JE. Vaccination with FimH adhesin protects cynomolgous monkeys from colonization and infection by uropathogenic Escherichia coli. J Infect Dis 2000;181:774-8.

26 Roberts JA, Kaack MB, Baskin G, et al. Antibody responses and protection from pyelonephritis following vaccination with purified Escherichia coli PapDG protein. J Urol 2004;171:1682-5. 